\title{
Problemáticas en torno de las ciberculturas. Una reflexión sobre las posibilidades y los límites de la etnografía virtual
}

\author{
Problems Surrounding Cybercultures. A Reflection \\ on the Possibilities and Limits of Virtual Ethnography
}

LIC. PROF. FEDERICO ÁLVAREZ GANDOLFI

UNIVERSIDAD DE BUENOS AIRES

Artículo recibido el / Article received: 01-11-2015

Artículo aceptado el / Article accepted: 05-09-2016

RESUMEN: Este artículo se propone presentar un acercamiento a los debates sobre la aplicación de técnicas propias del método etnográfico al estudio de comunidades que conforman y/o refuerzan lazos sociales en el ciberespacio. La necesidad de esta aproximación se debe a que, en Argentina, puede advertirse una escasa y dispersa producción académica respecto de las ciberculturas, aun dentro del contexto contemporáneo de creciente digitalización y convergencia donde las interacciones cotidianas vía Internet tienden a constituirse como una de las dimensiones centrales de la vida sociocultural. En consecuencia, aquí se propone una posible recopilación bibliográfica alrededor de las discusiones conceptuales y las reflexiones metodológicas que, tanto en la academia anglosajona como en la de habla hispana, se han dado desde la antropología, la sociología y la comunicación sobre lo que implica la «etnografía virtual» (Hine, 2004).

Palabras clave: ciberculturas, etnografía virtual, campo, ética.

ABSTRACT: The aim of this work is to present an approach to the discussions on the implementation of the ethnographic method to the study of community bonds in cyberspace. The need for such an approach is due to the fact that, in Argentina, it can be seen a sparse and scattered academic production regarding cybercultures, yet in the contemporary context of increasing digitization and convergence where everyday interactions via the Internet are established as one of the central dimensions of sociocultural life. Therefore, here is proposed a possible bibliography around the conceptual discussions and methodological reflections that, both in the Anglo and Hispanic academy, have been taking 
place from anthropology, sociology, and communication, about what "virtual ethnography" (Hine, 2004) implies.

Keywords: cybercultures, virtual ethnography, field, ethics.

\section{INTRODUCCIÓN ${ }^{1}$}

Para empezar a reflexionar sobre las implicaciones de una «etnografía virtual» (Hine, 2004), es necesario partir de algunas bases conceptuales. En primer lugar, puede entenderse que uno de los propósitos de la antropología consiste en el desarrollo de investigaciones sociales sobre el funcionamiento de comunidades (Hammersley y Atkinson, 1994 [1983]) o, en otros términos, en la comprensión de las lógicas culturales que estructuran las formas sociales de vinculación y diferenciación entre colectivos (Guber, 2009 [1991]). En segundo lugar, este análisis, a su vez, se sostiene en un método etnográfico a partir del cual se emprende una descripción densa de las prácticas habituales del colectivo de estudio y se explicitan e interpretan las estructuras de significación interiorizadas que las atraviesan, retomando los conocidos planteos de Clifford Geertz (2003 [1973]: 24).

Sin embargo, en las sociedades contemporáneas, la configuración de culturas o el tejido de vínculos comunitarios también pueden tener lugar a través de interacciones en el ciberespacio, lo que presenta nuevos desafíos analíticos. En este sentido, por ejemplo, partiendo de la conectividad rápida y global vía Internet habilitada por la digitalización, se profundizan las tensiones implicadas en los contactos interculturales que advierte Alejandro Grimson (2011). Así, pareciera que la exploración de los fenómenos socioculturales que tienen lugar en el ciberespacio es crucial para comprender el mundo contemporáneo de flujos transnacionales y fronteras simbólicas permeables. Una buena oportunidad para, finalmente, desplazar las nociones esencialistas de «la cultura»

1. El presente trabajo se realizó en el marco de una beca doctoral del Consejo Nacional de Investigaciones Científicas y Técnicas (CONICET). Asimismo, se inscribe dentro del proyecto de investigación acreditado UвACут (2015-2017) «Textos populares y prácticas plebeyas. Cultura de masas, género y fanatismos en la Argentina contemporánea», dirigido por el Dr. Pablo Alabarces y codirigido por la Dra. Libertad Borda, con sede en el Instituto de Investigaciones Gino Germani (IIGG) y financiado por la Secretaría de Ciencia y Técnica de la Facultad de Ciencias Sociales de la Universidad de Buenos Aires. Código 20020130100521BA. Área de Estudios Culturales. 
y «la identidad» como compartimentos estancos, internamente coherentes, homogéneos y atemporales, correspondientes a un territorio jurídico.

Es por ello que, como han observado otros autores (Welschinger Lascano, 2012), resulta al menos inquietante la escasa cantidad de trabajos académicos que pueden encontrarse en el ámbito local dedicados, desde un enfoque antropológico, a las interacciones vía Internet. En efecto, la ampliación de la mirada etnográfica hacia las ciberculturas o comunidades virtuales no debería resultar extraña, pues como señala Joan Mayans i Planells (2002: 81):

La etnografía se ocupó profusamente del Otro Distante antes de que una revolución tecnológica y la urbanidad contemporánea le empujaran a ocuparse también del Otro Próximo. La «revolución» digital, Internet y las [comunicaciones mediadas por ordenador] nos traen un regalo -quizá envenenadoen la forma del tele-otro o del ciber-otro (copresentes a distancia mediante tecnologías).

Tanto desde la sociología, los estudios culturales y las ciencias de la comunicación como desde la antropología, la implicación etnográfica ya ha demostrado ampliamente su riqueza para desarrollar comprensiones sobre culturas, comunidades o universos simbólicos «diferentes» en toda su complejidad. Esta riqueza reside en la presentación de análisis dialógicos desde el punto vista de los «nativos» (Ginsburg, 1989), en función de la reconstrucción de sus propios horizontes de sentido y mediante movimientos sucesivos de extrañamiento y familiarización, que permiten al investigador distanciarse de los propios presupuestos.

Aquí cabe recordar que, cuando la cultura investigada por el analista no forma parte de su cotidianeidad -abordaje del «Otro Distante»-, la aproximación física al campo permite transformar lo exótico en familiar, mientras que al estudiar su propia sociedad -abordaje del «Otro Próximo»- el etnógrafo busca convertir lo familiar en exótico. O como afirma Pablo Semán (2009: 190) basándose en la obra de Roberto DaMatta: «[1]a antropología capta la diferencia con lógicas contrapuestas: familiariza lo extraño y lo "salvaje" para incluirlo en lo humano, pero desfamiliariza y "extraña" lo próximo para no identificar su humanidad con nuestra versión de esta».

Ahora bien, ¿cómo podrían jugar estas observaciones en el despliegue de una práctica etnográfica en Internet? ¿Cómo ocuparse del «Tele o CiberOtro»? Cuando las comunidades de estudio se configuran a través de plataformas digitales, o los universos cuyas significaciones quieren analizarse son ciberculturas constituidas vía Internet, ¿es posible utilizar técnicas como la observación participante? ¿Dónde tendría lugar el trabajo de campo? ¿De qué 
modos podrían obtenerse informaciones y transformarlas en datos descriptivos a ser interpretados? ¿Cuáles serían las formas posibles de entrada en el campo y cumplimiento de estándares éticos?

Partiendo de estas preguntas, a continuación se presentará una síntesis de las principales discusiones que se han desarrollado en relación con las diferencias entre una etnografía clásica territorial y otra virtual en el ciberespacio. Dicha síntesis se centrará en problemáticas referidas al «campo» y a la ética del investigador, y estará acompañada por reflexiones personales surgidas a raíz de mi propia experiencia en el estudio de ciberculturas juveniles constituidas por «fans» argentinos de historietas y series animadas japonesas (Álvarez Gandolfi, 2014).

\section{LA PRÁCTICA ETNOGRÁFICA EN INTERNET}

En Argentina pueden encontrarse algunos trabajos que, desde disciplinas como la sociología, la comunicación y la sociosemiótica, señalan la importancia de abordar las interacciones vía Internet en toda su complejidad (Carlón y Scolari, 2012; Borda y Álvarez Gandolfi, 2014; Urresti et al., 2015). En este mismo sentido, aquí se propone rescatar la importancia del método cualitativo etnográfico para una descripción y comprensión profundas de las ciberculturas, también sugerida por Rosalía Winocur (2011).

En pos de subrayar dicha importancia, cabe recordar que el trabajo de campo implica dos técnicas fundamentales para producir un conocimiento no etnocéntrico: una observación participante del etnógrafo durante un período prolongado de tiempo y la realización de entrevistas en profundidad, no estructuradas ni directivas, o incluso el registro de conversaciones casuales. Ello permite un contacto directo con los «nativos», sobre cuya base puede captarse la riqueza de la diversidad social estudiada en función de la perspectiva de los actores (Guber, 2009 [1991]; Taylor y Bogdan, 1992).

A partir de estos principios etnográficos clásicos, Christine Hine (2004) apuesta por el desarrollo de una etnografía virtual y propone pensar a Internet como un ciberespacio donde se gestan diferentes culturas y formas de organización social, mediante interacciones sostenidas a través de los diversos usos cotidianos de las tecnologías digitales. Extrapolando los principios de la etnografía clásica, la ciberetnografía tendría que renunciar a tratar de entender Internet en su totalidad y, por lo tanto, ocuparse de distintos sitios web como casos particulares en torno de los cuales proponer generalizaciones mediante análisis comparativos (Miller y Slater, 2000). 
Entonces, en la medida en que pueden reconocerse ciertos consensos respecto del uso de la técnica de entrevista para el desarrollo de una etnografía virtual o ciberetnografía, ${ }^{2}$ en el siguiente apartado se desarrollarán problemáticas en relación con el ingreso al «campo» de trabajo y el desplazamiento del investigador dentro de él.

\section{EL PROBLEMA DE LA DELIMITACIÓN DEL CAMPO}

Hasta aquí se ha tratado de señalar la importancia de desarrollar estudios que, desde un enfoque socioantropológico, analicen las interacciones cotidianas que tienen lugar en el ciberespacio, en tanto estas se constituyen como una de las dimensiones centrales de la vida sociocultural contemporánea dados los acelerados avances tecnológicos. ${ }^{3}$ Una vez reconocida esta importancia, cabe preguntarse cómo pueden adaptarse las técnicas clásicas de la etnografía para realizar este tipo de abordajes de comunicaciones e intercambios simbólicos fluidos mediante plataformas digitales, sin referentes territoriales estables.

En este sentido, Hine (2004: 13) propone una metodología de investigación etnográfica para el estudio empírico de los usos cotidianos de Internet, retomando en principio los fundamentos tradicionales del método antes repasados:

En su forma básica, la etnografía consiste en que un investigador se sumerja en el mundo que estudia por un tiempo determinado y tome en cuenta las relaciones, actividades y significaciones que se forjan entre quienes participan en los procesos sociales de ese mundo. El objetivo es hacer explícitas ciertas formas de construir sentido de las personas, que suelen ser tácitas o que se dan por supuestas. El etnógrafo habita en una suerte de mundo intermedio, siendo simultáneamente un extraño y un nativo. Ha de acercarse suficientemente a la cultura que estudia como para entender cómo funciona, sin dejar de mantener la distancia necesaria para dar cuenta de ella.

2. La mayoría de los trabajos dedicados al despliegue de una práctica etnográfica en Internet coinciden en que las herramientas digitales pueden ser utilizadas para realizar entrevistas sincrónicas a distancia, aprovechando las ventajas de disponer de conversaciones textualizadas y transcriptas en el mismo momento que tienen lugar.

3. Cabe aclarar que la constitución de lo ciberespacial o de lo virtual como una de las dimensiones socioculturales centrales de la contemporaneidad no involucra solamente a sectores medios y altos, sino que se hace extensiva a los sectores populares urbanos (Urresti et al., 2015). 
El problema que conlleva esta afirmación consiste en las dificultades para delimitar dónde está el campo en el ciberespacio, es decir, en cómo saber dónde empieza y dónde termina ese «mundo» en el cual el investigador debe «sumergirse», cuando la red y los sitios presentes en ella parecieran ser infinitos e inabarcables. Respecto de esta problemática, una de las primeras advertencias que suelen hacer quienes desarrollan prácticas etnográficas en el ciberespacio radica en la necesidad de tomar conciencia de la deslocalización que lo caracteriza. Por ejemplo, Elisenda Ardèvol et al. (2008) subrayan la importancia de reconceptualizar el trabajo de campo etnográfico para adaptarlo a los nuevos entornos digitales en los que, a diferencia de lo que ocurre cuando se lleva adelante una etnografía clásica, el espacio sociocultural no se corresponde necesariamente con un espacio físico territorialmente acotado.

De aquí que, al no haber colocalización territorial ni interacciones cara a cara entre el investigador y los «nativos», pareciera que no es posible la aplicación del método etnográfico para el estudio de las ciberculturas o comunidades en línea que se forman a través de interacciones sociales vía Internet, mediadas por tecnologías digitales. Sin embargo, podría pensarse que la adaptación metodológica de las técnicas clásicas para realizar estudios antropológicos en el ciberespacio es necesaria, pues dichas tecnologías habilitan nuevos ámbitos de vinculación sociocultural entre los sujetos, potenciales informantes. Junto con las interacciones cara a cara, puede entenderse que los espacios virtuales se constituyen en «lugares» de intercambios simbólicos o campos de acción (Guber, 2009 [1991]), a los cuales también es preciso aproximarse.

Así, las principales técnicas etnográficas como la observación participante y la entrevista también podrían ser aplicadas al estudio de las formas de sociabilidad y configuración comunitaria que tienen lugar en la web. Es en esta misma dirección que Hine (2004) advierte la necesidad de problematizar uno de los fundamentos tradicionales del trabajo de campo antropológico: la necesidad de una copresencia física investigador-investigado, sostenida y prolongada en el campo de estudio, entendiendo la noción campo como un lugar o territorio delimitado donde se encuentran los actores miembros de la unidad sociocultural que se desea estudiar.

En el ciberespacio, las relaciones entre los propios sujetos de estudio -y los vínculos del investigador con ellos- parecieran no precisar del «cara a cara», pues tienen lugar dentro de Internet. De este modo, el despliegue de una «etnografía virtual» implicaría el seguimiento de las conexiones que se establecen a partir de los usos sociales de las tecnologías digitales, en función de 
la cibercultura que pretenda estudiarse y de los enlaces hipertextuales usados o promocionados por sus propios participantes.

Basándose en los planteos de George Marcus, Hine sugiere que la práctica etnográfica en Internet no tiene como condición un tradicional «estar ahí» -que ancla el relato descriptivo e interpretativo en la experiencia singular del analista, inmerso en un campo limitado territorialmente-, sino un «llegar ahí». Para ello, debe asumir un carácter multisituado, adaptativo, fluido, dinámico y móvil, además de ser acompañada por una flexibilidad interpretativa. Esta flexibilidad, a su vez, debe estar pendiente de la comprensión de los significados culturales que adquieren las múltiples conexiones establecidas desde la propia cibercultura o comunidad virtual investigada.

Entonces, el etnógrafo debe realizar inmersiones intermitentes en más de un entorno dentro de Internet, siguiendo las actividades y movimientos de la comunidad estudiada a través de varios sitios o plataformas de la red. Según mi propia experiencia, por ejemplo, para abordar las ciberculturas juveniles de «fans» argentinos de historietas y series animadas japonesas, el trabajo de campo puede comenzar en alguna de las plataformas digitales donde estos sujetos interactúan socialmente. Dichas plataformas son localizables mediante una búsqueda en la web, como páginas o grupos de Facebook (Álvarez Gandolfi, 2014), y deberían ser abordadas sobre la base de una explicitación de los criterios de selección para cada caso.

A partir de allí, podría ampliarse el campo a través de un seguimiento estratégico de los enlaces hipertextuales que se promuevan, los cuales pueden conducir a distintos sitios y a otras plataformas como YouTube o Instagram. La cantidad de ámbitos virtuales a incluir o el número de interacciones a observar podría depender del alcance de un punto de «saturación teórica» (Glaser y Strauss, 1967, citado en Taylor y Bogdan, 1992: 90), desde el cual se considere que las informaciones obtenidas ya no aportan elementos «nuevos» para el desarrollo de comprensiones.

Como afirma Hine, «[1]os límites [del «campo»] no son asunciones a priori, sino que se exploran en el curso de la etnografía» (2004: 81). Aquí habría una continuidad entre el trabajo de campo antropológico clásico y el «virtual», pues en ambos casos sus pautas se van construyendo a lo largo de la investigación, en función de la reflexividad de la relación entre los miembros de la situación de campo (Guber, 2009 [1991]). 


\section{EL PROBLEMA DE LA ÉTICA}

A las discusiones en torno de lo que implicaría la realización de una observación participante en el ciberespacio se les suma otra problemática central: cómo reflexionar sobre el lugar e involucramiento del investigador con respecto a la vida sociocultural de los sujetos entre los que estudia. Durante el desarrollo de una ciberetnografía o etnografía virtual, ¿cómo debe participar el investigador observador? ¿Bajo qué estándares éticos?

Cabe subrayar que la cuestión del equilibrio necesario entre observación y participación también se hace presente en la etnografía clásica. En efecto, Martyn Hammersley y Paul Atkinson ya afirmaron que la participación del etnógrafo puede ser «abierta o encubierta» (1994 [1983]: 1). En relación con dicho problema, Robert Kozinetz (2010) señala que varias investigaciones sobre ciberculturas o comunidades virtuales han optado por basarse en etnografías que son puramente observacionales. Si se piensa en términos de experiencia, tal decisión no debería sorprender, pues se corresponde con uno de los tipos de usuarios de tecnologías digitales que participan en interacciones vía Internet: el lurker o merodeador imperceptible. Este solamente observa un sitio web o plataforma digital sin presentarse ni comprometerse con la construcción y participación activas dentro de una comunidad virtual, evitando por ejemplo la publicación de mensajes.

No obstante, Kozinets afirma que «observar sin participar» no es ético, y subraya que la aproximación a las comunidades configuradas vía Internet debe basarse en la plena participación del etnógrafo como miembro de estas. Así, advierte que el investigador debe estudiar una comunidad cuyo acceso sea «público», presentarse ante los sujetos que participan de ella y comentarles sus objetivos, no revelar su identidad en sus trabajos, obtener su permiso para citar los mensajes que publican, mostrarles sus avances de investigación y pedirles devoluciones (2010: 136-156).

Respecto de estos planteos, podrían tomarse algunos reparos. La postura de Kozinets conlleva implícita cierta idealización de la presencia prolongada del etnógrafo en un espacio con límites bien definibles, fundamentos problematizados en el apartado anterior. Además, su hincapié en el hecho de que la comunidad estudiada sea «pública» podría ser discutible, en especial si se entiende que en el ciberespacio los límites entre «lo público» y «lo privado» se han vuelto difusos.

En este sentido, Joséph Walther (2002, citado en Estalella y Ardèvol, 2007) considera que el análisis de plataformas digitales equivale a «revisar una hemeroteca», por lo que no sería necesario cumplir con ningún estándar ético. 
Aquí puede reconocerse una influencia de Sherry Turkle (1995) y Howard Rheingold (1996), quienes sostienen que en Internet los individuos son libres para proyectar «yoes alternativos», dado que, protegidos por el anonimato y la ausencia de contactos físicos directos, pueden asumir múltiples identidades según sus propios deseos. Por lo tanto, uno no entraría en contacto con «personas humanas», sino con «efectos discursivos» reconstruibles en los textos producidos dentro del ciberespacio.

Sin pretensiones de caer en el determinismo tecnológico que atraviesa estas últimas ideas, podría pensarse que tanto la mirada de Kozinets como la de Walther son combinables. Por un lado, si se parte de las ideas desarrolladas por Libertad Borda (2012), la observación de plataformas digitales sin intervención encontraría una justificación aparentemente paradójica en uno de los principios clásicos de la etnografía: producir una aproximación a espacios donde se despliegan interacciones generadas de modo espontáneo entre los actores de una cultura, y no a instancias de un investigador. En un principio, la posición institucional con la que el analista suele ser asociado por los sujetos estudiados inhibe sus modos de vincularse, de modo que tampoco nada garantiza que los «nativos» contesten todas las preguntas del etnógrafo o que no actúen como suponen que este quiere que lo hagan. Dentro del ciberespacio, la protección de la pantalla y la anonimidad permitirían cierta desinhibición expresiva de los actores.

Por otro lado, tampoco hay que caer en lo que Rhiannon Bury (2005) llama "el sueño de la incorporeidad", según el cual dentro del ciberespacio no habría cuerpos sino solamente palabras, por lo que, gracias al «anonimato», cualquier usuario sería «libre» de asumir la identidad que desee en las interacciones vía Internet. Por el contrario, en la mayoría de las ciberculturas estructuradas en torno de intereses comunes, o al menos en aquellas vinculadas a algún tipo de fanatismo, el anonimato no es tal: los propios usuarios suelen percibir que las identidades «virtuales» puestas en escena en las comunicaciones entre pares tienden a coincidir con identidades personales «reales», ${ }^{4}$ en un movimiento de hacer público lo privado (Winocur, 2011). Esto responde a que, como se verá posteriormente, los contextos online y offline no son ámbitos de socialización escindidos, sino que se entrecruzan.

4. Aquí no se pretende señalar ningún tipo de juicio de valor en relación con «lo real»y «lo virtual», sino que se entiende, siguiendo a Marcelo Urresti et al. (2015), que en las sociedades contemporáneas tanto «la realidad» como «la virtualidad» son intercambiables entre sí, en términos de los sentidos concretos que les asignan las personas que desarrollan prácticas en ambas instancias. En general, los «encuentros virtuales» suelen ser complementados con «encuentros cara a cara». 
Por lo tanto, para cumplir con estándares éticos, el etnógrafo del ciberespacio podría asegurar la confidencialidad y preservar la identidad de los sujetos que observa, no publicando sus nombres ni imágenes correspondientes a sus participaciones en las plataformas digitales. En este sentido, durante el desarrollo de mi propia investigación, decidí no incluir impresiones de pantalla o capturas de los grupos de Facebook observados, sino que realicé transcripciones literales de las interacciones que tienen lugar allí y utilicé las iniciales de los nombres de perfil que sus participantes llevan dentro del sitio.

Para finalizar, el problema reside en que, aparentemente, si el analista opta por una observación de las interacciones en plataformas digitales sin intervenir en ellas, pasando desapercibido, estaría traicionando otro de los principios de la etnografía clásica: la posibilidad de que los sujetos investigados produzcan conocimientos sobre el etnógrafo de modo recíproco, central en el despliegue de una reflexividad sobre todo trabajo de campo. Sin embargo, en los espacios territoriales, la «diferencia» del observador también puede pasar desapercibida, y lo que le daría sustento «ético» a la investigación sería, precisamente, trabajar en profundidad con un grupo definido de asistentes, no con todos.

Entonces, del mismo modo en que a un etnógrafo que desarrollara una observación participante tradicional en partidos de fútbol, recitales de rock o locales bailables no se le cuestionaría el hecho de «no avisar» a la totalidad de concurrentes de que «están siendo observados» en ciertos momentos, tampoco podría cuestionársele a quien estudia interacciones vía Internet que no les haga ese aviso a todos los participantes del entorno virtual estudiado. En el ciberespacio, el analista podría partir de la observación sin intervención, para luego sí entrar en contacto con algunos de los actores y generar los respectivos consentimientos informados de modo de relacionarse con ellos como informantes clave.

\section{UN CIERRE A MODO DE APERTURA}

Si bien este trabajo se ha centrado en discusiones sobre las implicancias del desarrollo de una práctica etnográfica en Internet, ello no significa que el método virtual pueda prescindir completamente del método clásico. En efecto, la propuesta de Hine consiste en combinar un trabajo de campo en el ciberespacio con uno en espacios territoriales, es decir que no se limita la investigación al «delante de la pantalla» (Press y Livingstone, 2006), pues, en términos de la propia autora: 
Los usuarios de Internet dan sentido a sus prácticas a través de una comprensión compartida [y compleja] de la relación entre tecnología en Internet y las relaciones sociales en el tiempo y el espacio [...]. Más que trascender el tiempo y el espacio, Internet puede ser representada como una instancia de múltiples órdenes espaciales y temporales que cruzan una y otra vez la frontera entre lo online y lo offline (Hine, 2004: 21).

La inmersión del investigador en ámbitos de interacción offline o «presenciales», cara a cara, también permite dar cuenta de las maneras en que sus participantes los viven como instancias espaciotemporales que no son completamente ajenas a las de las interacciones online o «virtuales». Más bien, estas instancias presentan tanto divergencias como convergencias y ambas forman parte de la realidad cotidiana de los actores (Miller y Slater, 2000). De este modo, pueden describirse e interpretarse las formas en que los usos particulares de las tecnologías digitales adquieren significados dentro del contexto cultural más amplio donde tienen lugar.

Asimismo, dicha combinación de etnografías hace posible tomar distancia de las posturas tecnológico-deterministas señaladas anteriormente, pues permite advertir la persistencia en el ciberespacio de los clivajes de género, sexualidad, etnia, edad o clase como dimensiones de subalternidad. Estos clivajes no son anulados por una supuesta ausencia de prejuicios dada la incorporeidad o inexistencia de marcadores físicos, sino que pueden ser captados mediante marcas sociolingüísticas. Ello resulta más evidente al contemplar la dimensión performativa de las identidades culturales y las prácticas normativas a las que responde su puesta en escena.

Si bien, como anticipa Ana Laura Rivoir (1999), las interacciones vía Internet suelen ser leídas con una connotación positiva debido a sus pretendidas «horizontalidad», «flexibilidad», «solidaridad» y «pluralidad», algunos trabajos ya han intentado demostrar los distintos mecanismos a través de los cuales las desigualdades también se reproducen en diferentes entornos digitales (Bury, 2005; Álvarez Gandolfi, 2014).

Retomando las advertencias de Alejandro Grimson (2011), en lugar de pensar en términos de culturas o comunidades uniformes y con fronteras fijas, resulta más preciso encarar la práctica etnográfica teniendo en cuenta tanto las tramas simbólicas compartidas como las relaciones de desigualdad sedimentadas que puedan identificarse. Sobre todo en un contexto sociocultural contemporáneo en el cual «[c]uanto más se ha reducido la distancia física, cuanto más se ha intensificado la comunicación directa y massmediática, más han aumentado las distancias simbólicas, culturales e identitarias» (Grimson, 2011: 239). 
Entonces, ¿cómo podría desplegarse una práctica etnográfica en Internet? ¿De qué modos podrían adaptarse las técnicas tradicionales de la etnografía clásica? ¿Dónde empieza y dónde termina el trabajo de campo virtual? ¿Cuáles son los estándares éticos que deberían cumplirse? Estos son debates que quedan abiertos. Pero, en pos de afrontarlos, es necesario aceptar los desafíos implicados en el abordaje investigativo del ciberespacio, al cual la antropología tiene mucho que aportar.

Después de todo, uno de los objetivos principales del trabajo de campo etnográfico es «recabar material empírico», «reconstruir las lógicas propias del grupo social estudiado»y «reformular los propios modelos teóricos» (Guber, 2009 [1991]: 86). Y, para cumplir con estos objetivos en las sociedades contemporáneas, los estudios antropológicos no pueden ser renuentes a las ciberculturas, una de las nuevas formas posibles de vinculación social.

\section{REFERENCIAS BIBLIOGRÁFICAS}

Álvarez GANdolfi, F. (2014): Subcultura otaku. Representaciones, prácticas e identidades juveniles de los fans del manga y el anime en Argentina, Buenos Aires, tesis de Licenciatura en Ciencias de la Comunicación, Facultad de Ciencias Sociales, Universidad de Buenos Aires.

Ardèvol, E.; A. Estalella; D. Domínguez (2008): «Introducción: la mediación tecnológica en la práctica etnográfica» en Ardèvol, E.; A. EsTALELlA; D. Domínguez (coords.): La mediación tecnológica en la práctica etnográfica. Actas del XI Congreso de Antropología de la FAAEE, Donostia, Ankulegi, 9-29.

BORDA, L. (2012): Bettymaníacos, luzmarianas y mompirris: el fanatismo en los foros de telenovelas latinoamericanas, Buenos Aires, tesis de Doctorado en Ciencias Sociales, Facultad de Ciencias Sociales, Universidad de Buenos Aires.

BordA, L.; F. Álvarez Gandolfi (2014): "“Viejos criticando el anime": luchas simbólicas entre otakus y medios», Alaic. Revista Latinoamericana de Ciencias de la Comunicación, 21(11): 98-109.

Bury, R. (2005): Cyberspaces of Their Own, Nueva York, Peter Lang.

CARlón, M.; C. Scolari (comps.) (2012): Colabor_arte. Medios y arte en la era de la producción colaborativa, Buenos Aires, La Crujía.

Estalella, A.; E. Ardèvol (2007): «Ética de campo: hacia una ética situada para la investigación etnográfica de Internet», Forum: Qualitative Social Research, 3(8), septiembre [en línea]. Disponible en: http://www. 
qualitative-research.net/index.php/fqs/article/view/277/609, último acceso el 4 de septiembre de 2016.

GeERTZ, C. (2003 [1973]): La interpretación de las culturas, Barcelona, Gedisa.

GinsburG, F. (1989): «Cuando los nativos son nuestros vecinos» en BoIvin, M.; A. Rosato; V. Arribas (eds.): Constructores de otredad, Buenos Aires, Antropofagia, 246-254.

Grimson, A. (2011): Los límites de la cultura. Crítica de las teorías de la identidad, Buenos Aires, Siglo Veintiuno Editores.

GubER, R. (2009 [1991]): El salvaje metropolitano. Reconstrucción del conocimiento social en el trabajo de campo, Buenos Aires, Paidós.

Hammersley, M.; P. AtKinson (1994 [1983]): Etnografía. Métodos de investigación, Barcelona, Paidós.

Hine, C. (2004): Etnografía virtual, Barcelona, UOC.

Kozinetz, R. (2010): Netnography: Doing Ethnographic Research Online, Londres, Sage.

Mayans i Planells, J. (2002): «Nuevas tecnologías, viejas etnografías (objeto y método de la etnografía del ciberespacio)», Quaderns de l'Institut Català d'Antropologia, 17-18: 79-97. Disponible en: http://www.cibersociedad.net/archivo/articulo.php?art=23, último acceso el 4 de septiembre de 2016.

Miller, D.; D. Slater (2000): The Internet: an Ethnographic Approach, Oxford, Berg Publishers.

Press, A.; S. Livingstone (2006): «Taking audience research into the age of new media: old problems and new challenges» en White, M.; J. Schwoch (eds.): Questions of Method in Cultural Studies, Oxford, Blackwell, 175-200.

RHEINGOLD, H. (1996): La comunidad virtual, Barcelona, Gedisa.

RIvorR, A. L. (1999): «Redes sociales: ¿instrumento metodológico o categoría sociológica?», Revista de Ciencias Sociales, 15: 49-58. Disponible en: http://www.lasociedadcivil.org/wp-content/uploads/2014/11/articulo_redes.pdf, último acceso el 4 de septiembre de 2016.

SEMÁN, P. (2009): «Culturas populares: lo imprescindible de la desfamiliarización», Maguaré, 23: 181-205. Disponible en: http://www.bdigital.unal. edu.co/19030/1/14978-45191-1-Pв.pdf, último acceso el 4 de septiembre de 2016.

TaYlor, S.; R. Bogdan (1992): Introducción a los métodos cualitativos de investigación, Barcelona, Paidós. 
TuRkLe, S. (1995): Life on the Screen: Identity in the Age of the Internet, Nueva York, Paperback.

UrResti, M.; J. Linne; D. Basile (2015): Conexión total. Los jóvenes y la experiencia social en la era de la comunicación digital, Buenos Aires, Grupo Editor Universitario.

WelsChinger LASCANo, N. (2012): «La etnografía virtual revisitada: Internet y las nuevas tecnologías digitales como objeto de estudio», Revista Latinoamericana de Metodología de las Ciencias Sociales, 2(2): 109-116. Disponible en: http://sedici.unlp.edu.ar/bitstream/handle/10915/26949/ Documento_completo.pdf?sequence $=1$, último acceso el 4 de septiembre de 2016.

WinocuR, R. (2011): «Etnografía de un proceso de reconstrucción biográfica en Internet», Etnografías Contemporáneas, 5: 165-175. 